\title{
Diagnosing Celiac Disease: Role of the Pathologists
}

\author{
Marián Švajdler ${ }^{1,4, *}$, Ondřej Daum ${ }^{1,2}$, Boris Rychlý ${ }^{3}$ \\ ${ }^{1}$ Šikl’s Department of Pathology, Charles University, Medical Faculty in Pilsen, Czech Republic \\ ${ }^{2}$ Medical Faculty Hospital, Pilsen, Czech Republic \\ ${ }^{3}$ Cytopathos s.r.o., Bratislava, Slovakia \\ ${ }^{4}$ Louis Pasteur University Hospital, Košice, Slovakia \\ *Corresponding author: svajdler@yahoo.com
}

Received May 03, 2014; Revised June 02, 2014; Accepted June 06, 2014

\begin{abstract}
Duodenal biopsy is an essential component in the diagnosis of celiac disease (CD). Although the classical findings of increased number of intraepithelial lymphocytes, crypt hyperplasia and villous atrophy are very characteristic, the diagnosis cannot be achieved on the basis of histopathology alone, as there are many entities that can mimic CD and a close collaboration of a pathologist and a clinician specialist is needed. In a patient with suspected CD, pathologist should describe essential histopathological findings and offer differential diagnosis when appropriate. The most important recent changes in the diagnostic criteria for CD include lower numbers of intraepithelial lymphocytes that are considered to be normal and recommendation to perform biopsies from the proximal part of the duodenum in addition to the distal duodenal biopsies.
\end{abstract}

Keywords: celiac disease, histopathology, differential diagnosis

Cite This Article: Marián Švajdler, Ondřej Daum, and Boris Rychlý, "Diagnosing Celiac Disease: Role of the Pathologists.” International Journal of Celiac Disease, vol. 2, no. 2 (2014): 70-75. doi: 10.12691/ijcd-2-2-10.

\section{Introduction}

Small bowel mucosa reacts to numerous causes with only limited repertoire of morphological changes. Although mucosal changes in celiac disease (CD) are very typical, they are not disease specific, as there are many conditions that can mimic CD morphology. In a patient with suspected $C D$, pathologist should describe essential histopathological findings and offer differential diagnosis when appropriate. Final diagnosis should be based on a combination of clinical picture, serological testing, histology and a clinical and serologic response to a glutenfree diet. We will briefly discuss the histopathology of CD and its most important mimicking conditions. Recent advances in the definitions of the normal proximal small bowel intraepithelial lymphocytes (IEL) count will be reviewed first.

\section{Proximal Small Bowel IEL Count}

Increased number of IEL is considered to be the earliest and the most consistent change in CD [1]. Because more and more patients are biopsied with less severe symptoms or for screening purposes (e.g. latent CD patients with family history or positive serology only), many patients will have increased number of IEL as the sole abnormality (e.g. architecturally normal villi with raised IEL number). Until recently, the normal upper limit for small bowel IEL was 40 IEL/100 enterocytes. This limit value came from a study of jejunal capsule biopsies by Ferguson and Murray in early seventies [2]. However, jejunal biopsies are seldom used nowdays for diagnosis of CD, and more recent reports have defined the upper limits for duodenal mucosa as 20-25 IEL/100 enterocytes [3,4,5,6]. However, formal IEL counting in 100-1000 enterocytes is timeconsuming, impractical and probably not necessary in most cases. In normal small bowel mucosa, the number of IEL is greatest in the crypt epithelium and decreases gradually toward the tips of the villi in a "decrescendo pattern". In celiac disease, this “decrescendo pattern” is lost, because there is an increased number of IEL along the whole villous lenght, including the tips of the villi [7]. Counting the number of IEL per 20 enterocytes in five villous tips and looking for loss of the normal "decrescendo pattern" are considered to be useful, sensitive, and the most practical screening method for tentative diagnosis of CD $[5,8]$.

Some authors advocate use of immunohistochemistry (e.g. CD3 or CD8) to detect increased numbers of IEL [9]. Because of higher sensitivity of immunohistochemistry, when compared to hematoxylin and eosin-stained slides, values up to 25-29 CD3+ IEL/100 enterocytes have been proposed as normal [10], although lower number (e.g. 17 CD3+ IEL) was found in another recent study [6]. On the other hand, the routine use of immunohistochemistry is not recommended by others, because it can decrease sensitivity of observing the loss of "decrescendo pattern" [7] and in fact does not improve detection of glutensensitive enteropathy when hematoxylin and eosin-stained sections are normal [11].

\section{Untreated Celiac Disease}


Classical histopathological features of celiac disese include increased number of IEL, crypt hyperplasia, villous atrophy and and increased lamina propria inflammation (mostly lymphocytes, plasma cells and eosinophils, with few neutrophils). However; the spectrum of changes is highly variable, ranging from architecturally normal villi with raised IEL number only (discussed in the details above), to total villous atrophy with crypt hyperplasia [1]. Changes are described to be most severe in the proximal parts of the small bowel, with less severe changes in the distal parts. There is no strict association between clinical symptoms and the degree of histopathological changes in small bowell mucosa. Traditionally, it is believed that the lenght of small bowel with damaged mucosa rather than the degree of mucosal damage correlates with the clinical presentation. Thus, previously compensated and clinically silent mild degree of mucosal damage can be unmasked by an additional insult, for example infection, ischaemia or surgical shortening of the intestine $[1,7,12]$.

Exact number of tissue samples needed for the diagnosis of celiac disease is not strictly defined. American Gastroenterological Association (AGA) states that „it is important to take multiple endoscopic biopsy specimens (ideally $4-6$ biopsy specimens) from the proximal small intestine” [13], whereas North American Society for Pediatric Gastroenterology, Hepatology and Nutrition (NASPGHAN) recommends „multiple endoscopic biopsies be obtained from the more distal segments of the duodenum“ [14]. Because of the more widespread serological testing and higher clinical awareness, more patients are currently being diagnosed without classical symptoms (diarrhea, steatorrhea, weight loss, anemia), with much milder presentation (such as abdominal discomfort or flatulence), or without any symptoms $[15,16]$. In these cases, patchy distribution of mucosal changes can be expected, and more extensive bioptical sampling is recommended [17]. In general, samples with at least three or four well oriented villi and crypts are required for the diagnosis.

Interestingly, Drut and Rúa never observed patchiness of the disease or biopsies without villous atrophy in a large series of paediatric CD patients [18]. Because all patients in the series were symptomatic, these authors later performed a separate study in 10 children with positive antibodies for CD, but without any clinical evidence of malabsorption or growth retardation. Bioptical samples from the proximal (first and second) and distal (third and fourth) parts of duodenum were processed and evaluated separately. They showed in all patients advanced villous atrophy in the proximal duodenal mucosa and mild to absent involvement of the distal segments. They concluded that the presence of antibodies in the absence of malabsorption is always associated with mucosal damage, evident only in the proximal duodenum, and mucosal biopsy needs to be done in separate samples from proximal and distal duodenal mucosa [19]. More recent large scale studies in adult and pediatric patients confirmed that CD-related changes are always present in the duodenal bulb mucosa, and in some cases it is the only affected site [20,21]. All these studies suggest that in patients with suspected CD, optimal biopsy protocol should include duodenal bulb mucosa in addition to distal duodenum.
Crypt hyperplasia precedes villous atrophy in CD. As a result of crypt hyperplasia, overall thickness of mucosa does not change substantially despite villous atrophy [1]. The crypts contain stem cells, which are precursors of enterocytes and goblet cells during mucosal regeneration [12]. In untreated CD, the growth fraction of cells in the crypts, as measured by immunohistochemistry (MIB-1 antibody), is significantly higher when compared to treated CD cases or normal controls [22]. However, again, routine use of immunohistochemistry is neither needed, nor recommended.

Villous atrophy is considered to be the most typical finding in CD. However, the normal villous to crypt (V/C) ratio is still subject of controversy. Generally accepted normal range is $3: 1$ to $5: 1$ [23], but even ratio $1,22-2,46$ was reported as normal [3]. In children, $\mathrm{V} / \mathrm{C}$ ratio 2,5 or more is considered to be normal [18]. Because of these controversies, as well as missing recommendations on how to asses the V/C ratio (estimation or formal measurement), the evaluation of villous atrophy is highly subjective, especially of milder degrees.

Additional histological findings in CD, but with limited diagnostic values, are: (i) reduced height of enterocytes, (ii) pyknosis, (iii) loss of basal orientation and pseudostratification of the nuclei, (iv) reduced number of the goblet cells, and (v) reduction of the microvillous height $[12,14,22]$.

Importantly, diagnosis of CD and prescription of a gluten-free diet for life should not be made in the absence of compatible small intestinal histologic findings, irrespective of serology [13].

\section{Treated Celiac Disease and Follow-up Biopsies}

The clinical response to a gluten-free diet is rapid in most cases (days to weeks). However, it was shown that the histological recovery can take many months and is not complete in every patient despite strict diet [24,25,26]. In one study, only $74 \%$ of paediatric patients and only $17.5 \%$ of adult patients with good compliance and in a clinical and serological remission showed histologically normal mucosa in a follow-up biopsy after average period of two years [26]. Patients without normal histology showed increased numbers of IEL and/or mild villous atrophy. On the other hand, serological markers have relatively low negative predictive value in predicting mucosal damage in patients on a gluten-free diet. In a follow-up biopsy study of 87 adult patients, 27 showed villous atrophy (Marsh type 3 lesion, see later) despite the fact, that anti-tissue transglutaminase antibodies (tTG-Ab) were negative in 16 (59\%) [27]. Thus, the significance of persistent histological changes in the patients on a gluten-free diet is currently not entirely clear. Fortunately, remission of CD is currently defined clinically and serologically rather than pathologically: the aim is to abolish symptoms and normalize blood tests and serology. Regarding the followup biopsies, both AGA and NASPPGHAN now state that gluten challenge and a repeat biopsy are no longer required to establish the diagnosis of CD in patients whose initial small intestinal biopsy has the characteristic histological appearance and in whom an objective response to a gluten-free diet is obtained $[13,14]$. However, 
if the diagnosis is uncertain, or if symptoms persist despite the strict diet, other diseases should be excluded by a repeated biopsy, serology and HLA-DQ2 / HLA-DQ8 testing $[13,14,28]$.

Historically, small bowel enzymohistochemistry was a part of diagnostic workup in patients with CD. In fact, combined multiple sacharidases and peptidases deficiency is highly specific for CD [29]. Nowdays, it is not considered to be a routine investigation, except for cases, where a rare primary enyzme deficiency is in the differential diagnosis as a cause of malabsorption [30]. In an untreated CD, practically all patients have decreased activity of the whole spectrum of disacharidases and peptidases (mostly lactase and trehalase) [29] and glutenfree diet will restore the function of the enzymes. However, even after 9-19 months on a diet, activity of the enzymes is decreased and normalizes only after 24-48 months, except for lactase [25]. Thus, lactase enyzmohistochemistry in a follow-up biopsy of a symptomatic patient on a gluten free diet, can confirm clinically relevant persisten lactase defficiency.

\section{Histopathological Classification}

As mentioned earlier, the severity of mucosal changes in $\mathrm{CD}$ is very variable, ranging from almost normal to total villous atrophy [1]. Marsh proposed histopathological classification system [31,32], which after modification by Oberhuber et al. [33] is now widely used by pathologists. Marsh-Oberhuber classification includes five categories of lesions: type 0 (pre-infiltrative) with normal histology; type 1 (infiltrative) with increased IEL numbers only; type 2 with increased IEL and crypt hyperplasia (infiltrative-hyperplastic); type 3 (destructive) with increased IEL, crypt hyperplasia and villous atrophy (subclassified as 3a, 3b and 3c for mild, marked and total villous atrophy) and type 4 lesion (atrophic-hypoplastic) with increased IEL, normal crypt height and atrophic villi [33]. The most important aspect of the classification is that it helps pathologists to recognize the whole spectrum of changes, including the early infiltrative stage. However, it is only a non-mandatory supplement to the diagnosis, used mostly for research purposes (e.g. assesing regression of lesions after a gluten-free diet).

It is well known, that the greater number of categories is used in any classification, the lower is the intra- and interobserver reproducibility. Corazza and Villanacci therefore proposed simplified classification, but this classification did not come into general use so far [34]. A comparison of Marsh-Oberhuber classification and Corazza's classification is presented in the Table 1 . However, these authors have proposed that irreversible type 4 lesion (atrophic-hypoplastic) should be removed from the classification, because most of these patients present with refractory sprue (see later) and already have monoclonal IEL proliferation [34].

Table 1. A comparison of the Marsh-Oberhuber classification with the classification of Corraza et al

\begin{tabular}{|c|c|c|c|c|}
\hline Marsh-Oberhuber & Corazza & IEL & Crypts & Villi \\
\hline Type 0 & & $<40$ & Normal & Normal \\
\hline Type 1 & \multirow{2}{*}{ Grade A } & $>40$ & Normal & Normal \\
\hline Type 2 & & $>40$ & Hypertrophic & Normal \\
\hline Type 3а & \multirow{2}{*}{ Grade B1 } & \multirow{3}{*}{$>40$} & \multirow{3}{*}{ Hypertrophic } & Mild atrophy \\
\hline Type 3b & & & & Marked atrophy \\
\hline Type 3c & Grade B2 & & & Total atrophy \\
\hline Type 4 & deleted & $<40$ & Normal & Total atrophy \\
\hline
\end{tabular}

Alternative villous atrophy grading system for paediatric patients was also proposed by Drut and Rúa (Table 2) [18].

Table 2. Grading of villous atrophy according to Drut et al

\begin{tabular}{|c|c|c|}
\hline \multicolumn{2}{|c|}{ Villous atrophy } & Crypt to villous ratio \\
\hline \multicolumn{2}{|c|}{ Grade 1} & $2,5-2$ \\
\hline \multicolumn{2}{|c|}{ Grade 2} & $2-1$ \\
\hline Grade 3 & \multirow{2}{*}{ Always CD } & $1-0,5$ \\
\hline Grade 4 & & $<0,5$ \\
\hline
\end{tabular}

\section{Refractory CD}

Refractory CD (refractory sprue) is defined as "severe villous atrophy associated with severe malabsorption that either does not or no longer responds to a GFD” [13]. The diagnosis of RCD requires a combination of clinical and pathological findings, and the diagnosis is made on the basis of strong evidence of CD, supplemented with systematic exclusion of both other causes of non- responsive CD or villous atrophy and malignancy [35]. Non-compliance to the dietary restriction should be considered, including non-intentional gluten ingestion in contaminated food (a positive tTGA may reflect continued ingestion of gluten), as well as other causes of diarrhea or malabsorption, including (but not limited to) autoimmune enteropathy, common variable immunodeficiency syndrome, tropical sprue, collagenous sprue, microscopic colitis, small-bowel bacterial overgrowth, lactose intolerance, eosinophilic gastroenteritis, and Crohn's disease $[13,35]$. Once other diseases are ruled-out, the refractory CD can be classified into two types: Type I and type II. The type I refractory CD is characterized by IEL that have normal $\mathrm{CD}^{+} / \mathrm{CD}^{+}$phenotype and are polyclonal in the T-cell gene rearrangement studies. In contrast, majority of patients with either refractory CD or so called ulcerative jejunitis have clonal populations of IEL [36,37] and are now classified as type II refractory CD [35]. Although these phenotypically aberrant IEL are not morphologically recognizable as aberrant, nor they form tumor masses, they clearly represent risk and the first step in progression to the enteropathy-associated T-cell lymphoma (EATL) [36]. Because of poor prognosis of the 
type II refractory CD, the assistance of haematopathologist and appropriate immunohistochemical and molecular studies are recommended in clinically suspicious cases [35]. Interestingly, Verkarre et al. showed that $64 \%$ of patients with refractory CD had lymphocytic gastritis and 55\% had lymphocytic colitis. In all of these cases, the IEL showed aberrant phenotype (CD3 $\left.{ }^{+} / \mathrm{CD}^{-}\right)$ and 8/13 (62\%) samples from the stomach and 8/10 (80\%) samples from the colon showed monoclonal T-cell receptor $\gamma$ rearrangement [38]. Although prognostic significance of these findings is not entirely clear, this study suggests that the refractory CD of the second type may be a diffuse gastrointestinal disease and may be responsible for the rare occurence of EATL in the stomach, colon or outside the gastrointestinal tract.

\section{Histological Differential Diagnosis of Celiac Disease}

Increased number of IEL and villous atrophy are not specific for CD. These changes occur in multiple diseases and conditions and the following discussion is far from being complete. Only most important and common entities are mentioned briefly and summarised in the Table 3 .

Table 3. Conditions with increased IEL and/or villous atrophy and crypt hyperplasia that can mimic CD

\begin{tabular}{|l|l|}
\hline Helicobacter pylori infection & $\uparrow$ IEL \\
\hline Drugs & $\uparrow$ IEL (e.g. NSAIDs) + villous atrophy (e.g. olmesartan) \\
\hline Tropical sprue & Villous atrophy + crypt hyperplasia \\
\hline Giardia lamblia infection & \pm villous atrophy \\
\hline Bacterial overgrowth, other infections (e.g. parasitic) & $\uparrow$ IEL \pm villous atrophy \\
\hline Prolonged viral gastroenteritis & $\uparrow$ IEL + villous atrophy \\
\hline Other food allergies (e.g. cow's milk protein) & $\uparrow$ IEL \pm villous atrophy \\
\hline Autoimmune enteropathy & $\uparrow$ IEL + villous atrophy \pm crypt hyperplasia \\
\hline Extraintestinal autoimmune disorders & $\uparrow$ IEL \pm villous atrophy \\
\hline IgA deficiency and CVID & $\uparrow$ IEL + villous atrophy \\
\hline Peptic duodenitis & \pm villous atrophy \\
\hline Crohn's disease and ulcerative colitis & $\uparrow$ IEL + villous atrophy \\
\hline
\end{tabular}

个EL - increased number of intraepithelial lymphocytes

\pm - variable presence of changes.

Increased number of IEL in an architecturally normal duodenal mucosa can be found in patients with Helicobacter pylori gastritis and is reduced after antibiotic treatment [39]. Some drugs, such as nonsteroidal antiinflammatory drugs (NSAIDs) or proton pump inhibitors can cause increased IEL numbers, and some drugs are also capable of causing small intestinal villous atrophy (e.g. azathioprin, colchicine, ipilimumab, mycophenolate, NSAIDs, olmesartan) [40,41]. Tropical sprue is an endemic malabsorption syndrome with histology similar to $\mathrm{CD}$, although total villous atrophy is rare and changes are equally prominent in the jejunum and ileum in addition to the duodenum [42]. Infection by Giardia lamblia in most cases does not cause mucosal abnormalities, but variable villous atrophy can be found in a minority of cases $[42,43]$. Patients with bacterial overgrowth often show patchy villous blunting and variable increase of chronic inflammation reminiscent of CD [40,42]. Diffuse villous atrophy with increased IEL can also be seen in patients with prolonged viral gastroenteritis [7]. Other food allergies (such as cow's milk protein allergy) can be associated with increased IEL numbers [40,44] and sometimes with partial villous atrophy [17]. Autoimmune enteropathy (AIE) is a rare disease presenting with intractable diarrhea and microscopically characterized by subtotal to total villous atrophy, normal to slightly hyperplastic crypts with lymphocytic infiltration and moderate to marked chronic inflammation in the lamina propria. Foci of single-cell necrosis reminiscent of intestinal graft-versus host disease, depletion of mucous and Paneth cells and anti-enterocyte antibodies will distinguish AIE from CD [42,45]. Increased number of IEL and variable degree of villous atrophy were reported in patients with various extraintestinal autoimmune disorders, including

Hashimoto thyroiditis, Graves disease, rheumatoid arthritis, lupus erythomatosus, multiple sclerosis, psoriasis, ankylosing spondylitis or progressive systemic sclerosis $[40,46]$. Patients with IgA defficiency or common variable immunodeficiency (CVID) often have increased IEL and variable villous atrophy. In contrast to $\mathrm{CD}$, inflammatory infiltrate in the lamina propria is not as prominent as in CDand there can be reduced number of plasma cells in the lamina propria, and frequent infection (e.g. Giardia, Cytomegalovirus, Cryptosporidium) with acute or granulomatous inflammation and nodular lymphoid hyperplasia $[47,48]$. Peptic duodenitis is characterized by edema, acute inflammation in the lamina propria and epithelium, erosions, gastric (foveolar) metaplasia and variable villous atrophy. These changes typically occur in the proximal duodenum, but rarely can be found in the distal duodenum as well. Importantly, peptic duodenitis can co-exist with $\mathrm{CD}$, and can be distinguished by increased number of IEL [49,50]. Recent study showed that significant duodenal neutrophilia (including foci of cryptitis and crypt abscesses) is often found in patients with $\mathrm{CD}$, especially in the pediatric population (56\% of pediatric and $28 \%$ of adult CD patients), and is associated with more active disease [51]. Thus, the presence of neutrophils or foveolar metaplasia should not be used to rule-out the diagnosis of $\mathrm{CD}$. Patients with preactive Crohn's disease can show only increased numbers of IEL in the architecturally normal duodenal mucosa [7], while in florid stage there is a variable degree of architectural distortion, active inflamation with crypt abscesses, basal lymphoplasmacytosis, pyloric metaplasia and occasional granulomas [28]. Some patients with with ulcerative colitis showed diffuse chronic duodenitis, characterized by diffuse plasmacytosis in the lamina propria, patchy cryptitis, and variable blunting of villi [52]. As duodenal 
involvement in Crohn's disease and ulcerative colititis is seldom an isolated finding, clinical information and involvement of other parts of the gastrointestinal tract can be helpful in equivocal cases.

\section{Conclusions}

Duodenal biopsy is still an essential component in the diagnosis of $\mathrm{CD}$. Although the classical findings of increased number of IEL, crypt hyperplasia and villous atrophy are very characteristic, there are many entities that can mimic CD very closely. The diagnosis cannot be achieved on the basis of histopathology alone and a close collaboration of a pathologist and a clinician specialist is needed to evaluate every case. The most important recent changes in the diagnostic criteria for CD include (i) lower numbers of intraepithelial lymphocytes that are considered to be normal (20-25 IEL/100 enterocytes), (ii) recommendation to perform biopsies from the proximal part of the duodenum in addition to distal duodenal biopsies, and (iii) frequent finding of neutrophilic inflammation in CD.

\section{References}

[1] Marsh, M.N., Crowe, P.T, "Morphology of the mucosal lesion in gluten sensitivity," Baillieres Clin Gastroenterol, 9(2). 273-293. Jun.1995.

[2] Ferguson, A., Murray, D, "Quantitation of intraepithelial lymphocytes in human jejunum," Gut, 12(12). 988-994. Dec.1971.

[3] Hayat, M., Cairns, A., Dixon, M.F., O’Mahony, S, "Quantitation of intraepithelial lymphocytes in human duodenum: what is normal?," J Clin Pathol, 55(5). 393-394. May.2002.

[4] Mahadeva, S., Wyatt, J.I., Howdle, P.D, "Is a raised intraepithelial lymphocyte count with normal duodenal villous architecture clinically relevant?" J Clin Pathol, 55(6). 424-428. Jun.2002.

[5] Biagi, F., Luinetti, O., Campanella, J., Klersy, C., Zambelli, C., Villanacci, V., Lanzini, A., Corazza, G.R, "Intraepithelial lymphocytes in the villous tip: do they indicate potential coeliac disease?, " J Clin Pathol, 57(8). 835-839. Aug.2004.

[6] Pellegrino, S., Villanacci, V., Sansotta, N., Scarfi, R., Bassotti, G., Vieni, G., Princiotta, A., Sferlazzas, C., Magazzù, G., Tuccari, G, "Redefining the intraepithelial lymphocytes threshold to diagnose gluten sensitivity in patients with architecturally normal duodenal histology, " Aliment Pharmacol Ther, 33(6). 697-706. Mar.2011.

[7] Goldstein, N.S, "Proximal small-bowel mucosal villous intraepithelial lymphocytes," Histopathology, 44(3). 199-205. Mar.2004.

[8] Goldstein, N.S., Underhill, J, "Morphologic features suggestive of gluten sensitivity in architecturally normal duodenal biopsy specimens, " Am J Clin Pathol, 116(1). 63-71. Jul.2001.

[9] Mino, M., Lauwers, G.Y, "Role of Lymphocytic immunophenotyping in the diagnosis of gluten-sensitive enteropathy with preserved villous architecture," Am J Surg Pathol, 27(9). 1237-1242. Sep. 2003.

[10] Veress, B., Franzén, L., Bodin, L., Borch, K, "Duodenal intraepithelial lymphocyte-count revisited," Scand J Gastroenterol, 39(2). 138-144. Feb.2004.

[11] Hudacko, R., Zhou, X.K., Yantiss, R.K, "Immunohistochemical stains for CD3 and CD8 do not improve detection of glutensensitive enteropathy in duodenal biopsies," Mod Pathol, 26(9). 1241-1245. Sep.2013.

[12] Dickson, B.C., Streutker, C.J., Chetty, R, "Coeliac disease: an update for pathologists, " J Clin Pathol, 59(10). 1008-1016. Oct. 2006.

[13] Rostom, A., Murray, J.A., Kagnoff, M.F, "American Gastroenterological Association (AGA) Institute technical review on the diagnosis and management of celiac disease," Gastroenterology, 131(6). 1981-2002. Dec.2006.
[14] Hill, I.D., Dirks, M.H., Liptak, G.S., Colletti, R.B., Fasano, A., Guandalini, S., Hoffenberg, E.J., Horvath, K., Murray, J.A., Pivor, M., Seidman, E.G, "Guideline for the Diagnosis and Treatment of Celiac Disease in Children: Recommendations of the North American Society for Pediatric Gastroenterology, Hepatology and Nutrition, " J Pediatr Gastroenterol Nutr, 40(2). 1-19. Jan.2005.

[15] Ravikumara, M., Tuthill, D.P., Jenkins, H.R, "The changing clinical presentation of coeliac disease," Arch Dis Child, 91(12). 969-971. Dec.2006.

[16] van Heel, D.A., West, J, "Recent advances in coeliac disease, " Gut, 55(7). 1037-1046. Jul.2006.

[17] Lowichik, A., Book, L, "Pediatric celiac disease: clinicopathologic and genetic aspects, " Pediatr Dev Pathol, 6(6). 470-483. NovDec.2003.

[18] Drut, R., Rúa, E.C, "The histopathology of pediatric celiac disease: order must prevail out of chaos, " Int J Surg Pathol, 9(4). 261264.Oct.2001

[19] Drut, R., Rúa, E.C, "Histopathologic diagnosis of celiac disease in children without clinical evidence of malabsorption," Int J Surg Pathol, 15(4). 354-357. Oct.2007.

[20] Bonamico, M., Thanasi, E., Mariani, P., Nenna, R., Luparia, R.P., Barbera, C., Morra, I., Lerro, P., Guariso, G., De Giacomo, C., Scotta, S., Pontone, S., Carpino, F., Magliocca, F.M.; Società Italiana di Gastroenterologica, Epatologia, e Nutrizione Pediatrica, "Duodenal bulb biopsies in celiac disease: a multicenter study, " $J$ Pediatr Gastroenterol Nutr, 47(5). 618-622. Nov.2008.

[21] Evans, K.E., Aziz, I., Cross, S.S., Sahota, G.R., Hopper, A.D., Hadjivassiliou, M., Sanders, D.S, "A prospective study of duodenal bulb biopsy in newly diagnosed and established adult celiac disease," Am J Gastroenterol, 106(10). 1837-1842. Oct.2011

[22] Przemioslo, R., Wright, N.A., Elia, G., Ciclitira, P.J, "Analysis of crypt cell proliferation in coeliac disease using MI-B 1 antibody shows an increase in growth fraction," Gut, 36(1). 22-27. Jan.1995.

[23] Segal, G.H., Petras, R.E, Small intestine. In: Sternberg SS, ed. Histology for pathologists, Raven Press, New York, 1992, 547572.

[24] Rosekrans, P.C., Meijer, C.J., Polanco, I., Mearin, M.L., van der Wal, A.M., Lindeman, J, "Long-term morphological and immunohistochemical observations on biopsy specimens of small intestine from children with gluten-sensitive enteropathy, " J Clin Pathol, 34(2). 138-144. Feb.1981.

[25] Grefte, J.M. Bouman, J.G., Grond, J., Jansen, W., Kleibeuker, J.H, "Slow and incomplete histological and functional recovery in adult gluten sensitive enteropathy," J Clin Pathol, 41(8). 886-891. Aug.1988.

[26] Bardella, M.T., Velio, P., Cesana, B.M., Prampolini, L., Casella, G., Di Bella, C., Lanzini, A., Gambarotti, M., Bassotti, G., Villanacci, V," Coeliac disease: a histological follow-up study, " Histopathology, 50(4). 465-467. Mar.2007.

[27] Kaukinen, K., Sulkanen, S., Maki, M., Collin, P, "IgA class transglutaminase antibodies in evaluating the efficacy of glutenfree diet in coeliac disease," Eur J Gastroenterol Hepatol, 14(3). 311-315. Mar.2002.

[28] Pai R.K, "A practical approach to small bowel biopsy interpretation: Celiac disease and its mimics, " Semin Diagn Pathol, 31(2). 124-136. Mar.2014.

[29] Mercer, J., Eagles, M.E.,Talbot, I.C, "Brush border enzymes in coeliac disease: histochemical evaluation," J Clin Pathol, 43(4). 307-3012. Apr.1990.

[30] Lowichik, A., Book, L, "Pediatric celiac disease: clinicopathologic and genetic aspects, "Pediatr Dev Pathol, 6(6). 470-483. NovDec. 2003.

[31] Marsh, M.N, "Grains of truth: evolutionary changes in small intestinal mucosa in response to environmental antigen challenge" Gut, 31(1). 111-114. Jan. 1990.

[32] Marsh, M.N, "Gluten, major histocompatibility complex and the small intestine. A molecular and immunobiologic approach to the spectrum of gluten sensitivity (celiac sprue), "Gastroenterology, 102(1). 330-354. Jan.1992.

[33] Oberhuber, G., Granditsch, G., Vogelsang, H, "The histopathology of coeliac disease: time for a standardized report scheme for pathologists, " Eur J Gastroenterol Hepatol, 11(10). 1185-1194. Oct. 1999.

[34] Corazza, G.R., Villanacci, V, "Coeliac disease, "J Clin Pathol, 58(6). 573-574. Jun.2005.

[35] Rubio-Tapia, A., Murray, J.A, "Classification and management of refractory coeliac disease," Gut, 59(4). 547-557. Apr.2010. 
[36] Bagdi, E., Diss, T.C., Munson, P., Isaacson, P.G, "Mucosal intraepithelial lymphocytes in enteropathy-associated T-cell lymphoma, ulcerative jejunitis, and refractory celiac disease constitute a neoplastic population," Blood; 94(1). 260-264. Jul.1999.

[37] Daum, S., Weiss, D., Hummel, M., Ullrich, R., Heise, W., Stein, H., Riecken E.O., Foss, H.D.; the Intestinal Lymphoma Study Group, "Frequency of clonal intraepithelial $\mathrm{T}$ lymphocyte proliferations in enteropathy-type intestinal $\mathrm{T}$ cell lymphoma, coeliac disease, and refractory sprue," Gut, 49(6). 804-812. Dec.2001.

[38] Verkarre, V., Asnafi, V., Lecomte, T., Patey Mariaud-de Serre, N., Leborgne, M., Grosdidier, E., Le Bihan, C., Macintyre, E., Cellier, C., Cerf-Bensussan, N., Brousse, N, "Refractory coeliac sprue is a diffuse gastrointestinal disease, " Gut, 52(2). 205-211. Feb.2003.

[39] Nahon, S., Patey-Mariaud De Serre, N., Lejeune, O., Huchet, F.X., Lahmek, P., Lesgourgues, B., Traissac, L., Bodiguel, V., Adotti, F., Tuszynski, T., Delas, N, "Duodenal intraepithelial lymphocytosis during Helicobacter pylori infection is reduced by antibiotic treatment" Histopathology, 48(4). 417-423. Mar.2006.

[40] Brown, I., Mino-Kenudson, M., Deshpande, V., Lauwers, G.Y, "Intraepithelial lymphocytosis in architecturally preserved proximal small intestinal mucosa. An increasing diagnostic problem with a wide differential diagnosis," Arch Pathol Lab Med, 130(7). 1020-1025. Jul. 2006.

[41] De Petris, G., Caldero, S.G., Chen, L., Xiao, S.Y., Dhungel, B.M., Spizcka, A.J., Lam-Himlin, D, "Histopathological changes in the gastrointestinal tract due to medications: an update for the surgical pathologist (part II of II)," Int J Surg Pathol, 22(3). 202-211. May.2014.

[42] Owens, S.R., Greenson, J.K, "The pathology of malabsorption: current concepts," Histopathology, 50(1). 64-82. Jan.2007.

[43] Oberhuber, G., Stolte, M, "Giardiasis: analysis of histological changes in biopsy specimens of 80 patients," J Clin Pathol, 43(8). 641-643. Aug.1990.
[44] Augustin, M., Karttunen, T., Kokkonen, J, "TIA1 and mast cell tryptase in food allergy of children: increase of intraepithelial lymphocytes expresing TIA1 associates with allergy," J Pediatr Gastroenterol Nutr, 32(1). 11-18. Jan.2001.

[45] Russo, P.A., Brochu, P., Seidman, E.G., Roy, C.C, "Autoimmune enteropathy," Pediatr Dev Pathol, 2(1). 65-71. Jan-Feb.1999.

[46] Kakar, S., Nehra, V., Murray, J.A., Dayharsh, G.A., Burgart, L.J, "Significance of intraepithelial lymphocytosis in small bowel biopsy samples with normal mucosal architecture," $\mathrm{Am} J$ Gastroenterol, 98(9). 2027-2033. Sep.2003.

[47] Washington, K., Stenzel, T.T., Buckley, R.H., Gottfried, M.R, "Gastrointestinal pathology in patients with common variable immunodeficiency and X linked agammaglobulinaemia," Am J Surg Pathol,.20(10). 1240-1252. Oct.1996.

[48] Daniels, J.A., Lederman, H.M., Maitra, A., Montgomery, E.A, "Gastrointestinal tract pathology in patients with common variable immunodeficiency (CVID): a clinicopathologic study and review", Am J Surg Pathol, 31(12). 1800-1812. Dec.2007.

[49] Jeffers, M.D., Hourihane, D.O, "Coeliac disease with histological features of peptic duodenitis: Value of assessment of intraepithelial lymphocytes," J Clin Pathol, 46(5). 420-424. May.1993.

[50] Leonard, N., Feighery, C.F., Hourihane, D.O, "Peptic duodenitisdoes it exist in the second part of the duodenum?," J Clin Pathol, 50(1). 54-58. Jan.1997.

[51] Moran, C.J., Kolman, O.K., Russell, G.J., Brown, I.S., MinoKenudson, M, "Neutrophilic infiltration in gluten-sensitive enteropathy is neither uncommon nor insignificant: assessment of duodenal biopsies from 267 pediatric and adult patients," Am J Surg Pathol, 36(9). 1339-1345. Sep.2012.

[52] Lin, J., McKenna, B.J., Appelman, H.D, "Morphologicfindings in upper gastrointestinal biopsies of patients with ulcerative colitis: a controlled study,” Am J Surg Pathol, 34(11).1672-1677. Nov.2010. 